\title{
EDITORIAL
}

\section{Immediate Surgery for the Treatment of Open Angle Glaucoma?}

Before von Graefe introduced iridectomy and Critchett iridesis, both in 1857 the diagnosis of glaucoma meant ultimate blindness. Eserine (Physostigamine) was used by Laqueur in 1876 to induce miosis of the pupil. He also found that it reduced the intraocular pressure in glaucomatous patients but because it was so uncomfortable to use Weber tried Pilocarpine in 1877. Since then there has been continuing controversy as to when or whether surgical or medical therapy should be used for the treatment of open angle glaucoma. As always when a new method of treatment is introduced the pendulum swings in that direction; the most recent being the locally applied B blockers, laser therapy and trabeculectomy.

Mackenzie in Glasgow in 1830 is said to have suggested sclerotomy for the treatment of glaucoma. Paracentesis he certainly undertook but it is unclear whether the sclerotomy operation was ever performed. However, in this issue Jay and Allan present the latest practical information from that same city on a prospective, randomised, multicentre trial in which patients with glaucoma were either treated immediately by trabeculectomy (and all but four patients agreed to this) or by what is currently regarded as the conventional treatment with eye drops. Only if these failed to control the pressure or visual field did these patients have surgery.

This study, which has now been running for eight years (mean 4.6 years) show that of those who had surgery immediately after diagnosis $90 \%$ had no field change three years after the surgery whereas only $60 \%$ of those who had medical treatment had no change in the visual field. Furthermore, the loss of visual field in the medically treated group occurred in the first two years after diagnosis whilst the treatment was being adjusted or the decision to operate was made, as indeed it was in more than half of the whole group. However, once control was achieved the diminution in visual field remained the same; those with severe visual field loss continuing to lose this field gradually in spite of the normalisation of pressure, and those with little field loss not losing anything more.

Procrastination must now be regarded as a real evil in the treatment of open angle glaucoma. It is clear that it is safe and practical to treat about a quarter of the patients medically. The rest (unless the diode laser proves more successful than Argon) are going to need surgical treatment. As most of the field loss even in the moderately well controlled occurs within the first year the patients are going to need to be seen frequently during this year. The authors also point out that the vision is not compromised by early surgery and give guidelines as to those who need early surgery (IOP $>25$ and dense 1-4e Goldmann scotomas) and those who can be given a trial of medical therapy (1-2e Goldmann scotomas). 'Maximal medical therapy' no longer has a legitimate role in the management of uncomplicated open angle glaucoma and it seems to be emerging that 'minimal medical therapy' has also only a limited role to play.

The results found in this trial will require a major change of thinking on the part of many ophthalmologists and will have considerable consequences in the number of outpatient attendances, the staff to do the investigations and the surgical procedures which will be necessary. Nevertheless, this nettle needs to be grasped to ensure that those with this very unpleasant disease do not continue to lose vision.

P. G. Watson 